Influence of molecular vibrations on the valence electron momentum distributions of adamantane

Peer-reviewed author version

MORINI, Filippo; Watanabe, Noboru; Kojima, Masataka; DELEUZE, Michael \& Takahashi, Masahiko (2017) Influence of molecular vibrations on the valence electron momentum distributions of adamantane. In: JOURNAL OF CHEMICAL PHYSICS, 146(9) (Art N 094307).

DOI: $10.1063 / 1.4977060$

Handle: http://hdl.handle.net/1942/23319 


\title{
Influence of molecular vibrations on the valence electron momentum distributions of adamantane
}

Filippo Morini, ${ }^{1,}$ a) Noboru Watanabe, ${ }^{2}$ Masataka Kojima, ${ }^{2}$ Michael Simon Deleuze, ${ }^{1}$ and

$$
\text { Masahiko Takahashi }{ }^{2}
$$

${ }^{1}$ Center of Molecular and Materials Modelling, Hasselt University, Agoralaan Gebouw D, B-3590 Diepenbeek, Belgium

${ }^{2}$ Institute of Multidisciplinary Research for Advanced Materials, Tohoku University, Sendai 9808577, Japan

\begin{abstract}
We report an electron momentum spectroscopy study of vibrational effects on the electron momentum distributions of the outer valence orbitals of adamantane $\left(\mathrm{C}_{10} \mathrm{H}_{16}\right)$. The symmetric noncoplanar (e, 2e) experiment has been carried out at an incident electron energy of 1.2 keV. Furthermore, theoretical calculations of the electron momentum distributions with vibrational effects being involved have been performed using the harmonic analytical quantum mechanical and Born-Oppenheimer molecular dynamics approaches. In spite of the complex nature of the vibrational structure of this large molecule, both approaches provide overall quantitative insights into the results of the experiment. Comparisons between experiment and theory have shown that ground state nuclear dynamics appreciably affects the momentum profiles of the $7 \mathrm{t}_{2},\left\{2 \mathrm{t}_{1}+3 \mathrm{e}\right\}$, and $\left\{5 t_{2}+5 a_{1}\right\}$ orbitals. It has been demonstrated that changes in the momentum profiles are mainly due to the vibrational motions associated with the $\mathrm{CH}$ bonds.
\end{abstract}

Keywords: Electron Momentum Spectroscopy, Born-Oppenheimer Molecular Dynamics, Molecular Vibrations, Momentum Space Quantum Mechanics, Density Functional Theory

a) Corresponding author: filippo.morini@uhasselt.be 


\section{INTRODUCTION}

Diamondoids, ${ }^{1,2}$ hydrocarbons with diamond cage structures, exhibit both diamond-like properties and nanosize effects. The unusual electronic properties of these molecules make them attractive for widespread nanotechnological applications, such as electronic and opto-electronic devices. For this reason, diamondoids have been the subjects of a number of experimental and theoretical studies. ${ }^{3-10}$ In particular, the electronic structure of the smallest diamondoid, adamantane $\left(\mathrm{C}_{10} \mathrm{H}_{16}\right)$, has been thoroughly investigated so far because of its fundamental importance. ${ }^{11-21}$

Recent theoretical studies have shown that the quantum dynamics of nuclei is key to understanding the electronic and optical properties of diamondoids. ${ }^{6-10} A b$ initio calculations taking nuclear dynamics into account have revealed that the interplay between electronic and nuclear motions drastically affects the lineshapes of the absorption bands and the optical gap, ${ }^{7}$ and also shown that the molecular vibration play important roles in ionization processes of the molecules. ${ }^{10}$

Electron momentum spectroscopy (EMS), ${ }^{22-28}$ also known as binary (e, 2e) spectroscopy, may offer an opportunity to examine such influences. The basis of the EMS technique is the observation of electron impact ionization reactions under the high-energy Bethe-ridge conditions. Within the plane-wave impulse approximation (PWIA), the EMS cross section is directly related to the electron momentum density distributions or electron momentum profiles of the ionized orbital. ${ }^{25-28}$ Because of its unique ability to image individual electron orbitals in momentum space, EMS can provide a powerful means to investigate how molecular orbital patterns are affected by the vibrational motions of molecules. Indeed, recent EMS studies on various polyatomic molecules ${ }^{29-38}$ have shown that electron momentum profiles may considerably be affected by molecular vibrations, and that EMS experiments in conjunction with recently developed theoretical approaches are capable of disentangling the interplay between the electronic and nuclear dynamics. ${ }^{30,31,34-38}$ It is therefore of interest to apply the EMS technique to the study of the nuclear dynamics effects for diamondoids. 
In this paper we report an EMS study on the smallest diamond cage, adamantane. An EMS measurement on adamantane was already reported by Litvinyuk et al. in $2000,{ }^{39}$ but no attempt so far has been made to examine vibrational effects on the electron momentum profiles of this molecule. The aim of the present study is thus to elucidate the influence of nuclear dynamics on the electron momentum density distributions. For the purpose, the present experiment has been carried out by using one of our highly sensitive EMS spectrometers. ${ }^{40}$ Considerable improvement in the statistical accuracy of data has been achieved as compared with that of the study by Litvinyuk et al. Furthermore, theoretical calculations using two complementary approaches have been carried out to analyze vibrational effects on electron momentum profiles. The first of these, known as the harmonic analytical quantum mechanical (HAQM) method, ${ }^{30,35}$ is based on the harmonic approximation and the second approach relies on Born-Oppenheimer molecular dynamics (BOMD). ${ }^{41-43}$

\section{EXPERIMENT}

EMS is a high-energy electron-impact ionization experiment in which the inelastically scattered and ejected electrons are detected in coincidence. The binding energy, $E_{\text {bind }}$, and the momentum of the target electron before ionization, $\boldsymbol{p}$, can be determined using the following conservation laws of energy and momentum:

$$
\begin{gathered}
E_{\text {bind }}=E_{0}-E_{1}-E_{2}, \\
\boldsymbol{p}=\boldsymbol{p}_{1}+\boldsymbol{p}_{2}-\boldsymbol{p}_{0} .
\end{gathered}
$$

Here $E_{j}$ 's and $\boldsymbol{p}_{j}$ 's $(j=0,1,2)$ denote the kinetic energies and momenta of the incident, inelastically scattered, and ejected electrons, respectively.

The EMS experiment on adamantane was conducted at $E_{0}=1.2 \mathrm{keV}$ in the symmetric noncoplanar geometry, where the two outgoing electrons having equal energies $\left(E_{1}=E_{2}\right)$ and equal scattering polar angles of $45^{\circ}$ are detected in coincidence. In this kinematics, the magnitude of the target electron momentum, $p=|p|$, is straightforwardly related to the out-of-plane azimuthal angle 
difference between the two outgoing electrons $\left(\Delta \phi=\phi_{2}-\phi_{1}-\pi\right)$. The EMS spectrometer used in this study has been described in detail elsewhere. ${ }^{40}$

The experimental result was obtained by accumulating data at an ambient sample pressure of 1.6 $\times 10^{-4} \mathrm{~Pa}$ for three weeks runtime. Commercially available adamantane (Wako Pure Chemical Industries Ltd., $>99.0 \%$ ) was used in the experiment. During the measurement the electron gun produced an electron beam of typically $65 \mu \mathrm{A}$. The resulting instrumental energy- and momentumresolutions were $1.7 \mathrm{eV}$ full width at half maximum (FWHM) and about 0.15 a. u. at $p=1.0$ a. u., respectively.

\section{THEORETICAL CALCULATIONS}

Two different but complementary theoretical methods, which are the so-called HAQM and BOMD approaches, are currently used to calculate the momentum profiles with vibrational effects being involved. The HAQM approach treats nuclear motions quantum mechanically within the framework of the harmonic oscillator approximation. Owing to the approximation used, however, it does not account for non-harmonic effects, such as anharmonicities in the underlying potential energies, couplings between vibrations and rotations, and so on. Such effects can be taken into account by the BOMD approach, while it treats nuclear motions classically.

Since the theory behind the HAQM approach has been fully described elsewhere, ${ }^{30,34,35}$ only a short account is given here. Under the assumption of the PWIA the EMS cross section can be written as

$$
\sigma_{\mathrm{EMS}}=(2 \pi)^{4} \frac{p_{1} p_{2}}{p_{0}} f_{\mathrm{ee}} M(p)
$$

where $f_{\mathrm{ee}}$ is the electron-electron collision factor and $M(p)$ is the structure factor corresponding to a momentum profile. $^{23}$ In the frame of the Born-Oppenheimer approximation, the electron momentum profile for a transition from the initial neutral electronic state $\mathrm{i}$ to the final ion state $\mathrm{f}$ can be expressed as follows: ${ }^{30,34,35}$ 


$$
M(p)=\int\left[\sum_{\mathrm{v}} P_{\mathrm{v}}(T)\left|\chi_{\mathrm{iv}}(\boldsymbol{Q})\right|^{2}\right] \rho_{\mathrm{f}}(p, \boldsymbol{Q}) d \boldsymbol{Q}
$$

where $\chi_{\text {iv }}(\boldsymbol{Q})$ is the wave function of the v vibrational level in the initial electronic state and $P_{\mathrm{v}}(T)$ denotes the related Boltzmann's statistical population at temperature $T . \rho_{\mathrm{f}}(p, \boldsymbol{Q})$ represents a spherically-averaged electron momentum density distribution at a given molecular geometry, $\boldsymbol{Q}$.

$$
\rho_{\mathrm{f}}(p, \mathbf{Q})=\frac{1}{4 \pi} S_{\mathrm{f}}(\mathbf{Q}) d_{\mathrm{f}} \int\left|\psi_{\mathrm{f}}(\boldsymbol{p} ; \mathbf{Q})\right|^{2} d \Omega_{\mathbf{p}}
$$

Here $\psi_{\mathrm{f}}(\boldsymbol{p})$ denotes the momentum space representation of the normalized Dyson orbital and $S_{\mathrm{f}}$ the corresponding spectroscopic factor. $d_{\mathrm{f}}$ implies the degeneracy of the orbital and $(4 \pi)^{-1} \int \mathrm{d} \Omega_{p}$ represents the spherical averaging due to the random orientation of the molecule in gas phase.

In the HAQM approach, $\chi_{\mathrm{iv}}(\boldsymbol{Q})$ is described as a product of harmonic oscillator functions $\xi_{\mathrm{vL}}\left(Q_{\mathrm{L}}\right)$ with $Q_{\mathrm{L}}$ being the normal coordinate of the L-th vibrational mode, and it is further assumed that $\rho_{\mathrm{f}}(p, \boldsymbol{Q})$ changes slowly with $\boldsymbol{Q}$ in the neighborhood of $\boldsymbol{Q}_{0}$. As a result, the following computationally tractable expression is obtained:

$$
M(p)=\frac{1}{4 \pi} S_{\mathrm{f}}\left(\boldsymbol{Q}_{0}\right) d_{\mathrm{f}} \int\left|\varphi_{\mathrm{f}}\left(\boldsymbol{p}, \boldsymbol{Q}_{0}\right)\right|^{2} d \Omega_{p}+\sum_{\mathrm{v}} P_{\mathrm{v}}(T) d_{\mathrm{f}} \sum_{\mathrm{L}}\left\langle\xi_{\mathrm{vL}}\left|\rho_{\mathrm{f}}\left(p, \boldsymbol{Q}_{0}+Q_{\mathrm{L}} \hat{\boldsymbol{q}}_{\mathrm{L}}\right)-\rho_{\mathrm{f}}\left(p, \boldsymbol{Q}_{0}\right)\right| \xi_{\mathrm{vL}}\right\rangle,
$$

where $\hat{\boldsymbol{q}}_{L}$ denotes a unit vector that points along the L-th normal coordinate, and with $P_{V}(T)$ being the Boltzmann's statistical population of the vibrational level $v$ at temperature $T$. The first term is equivalent to the momentum profile for the molecule at the equilibrium geometry and the second term represents vibrational effects. The advantage of this method is not only in reducing the computational cost, compared to the calculation over the full $\boldsymbol{Q}$ space according to Eq. (4), but also in the ability to discuss vibrational effects by dividing those into contributions from each normal mode, as will be discussed later.

The HAQM results have been obtained according to the following procedure. First, the vibrational normal coordinates and frequencies were calculated for the electronic ground state by means of Density Functional Theory (DFT) along with the Becke-3-parameters-Lee-Yang-Parr (B3LYP) functional ${ }^{44,45}$ and Dunning's augmented correlation-consistent polarization valence basis 
set of double-zeta quality (aug-cc-pVDZ). ${ }^{46,47}$ The vibrational frequencies thus obtained using the Gaussian03 program ${ }^{48}$ are presented in Table I, showing a good agreement with the available experimental results. ${ }^{49,50}$

Subsequently, $\rho_{\mathrm{f}}(p, \boldsymbol{Q})$ 's were computed within the target Kohn-Sham approximation ${ }^{51}$ at several nuclear geometries distorted from equilibrium along each normal coordinate. For each distorted structure, the Kohn-Sham orbitals were obtained on the ground of DFT calculations in conjunction with the B3LYP functional and aug-cc-pVDZ basis set using the Gaussian03 program. The orbitals were subsequently converted to $\rho_{\mathrm{f}}(p, \boldsymbol{Q})$ 's with the aid of the MOMAP program developed by Brion and his co-workers, ${ }^{52}$ assuming a constant spectroscopic strengths for the orbitals under consideration. This assumption is proven by many studies of ionization spectra performed using Green's Function Theory $[\mathrm{ADC}(3)]$ or SAC-CI ${ }^{15,53-56}$ on saturated compounds, showing that $S_{\mathrm{f}}(\boldsymbol{Q}) \sim$ 1 for valence orbitals. Finally, the momentum profiles were obtained assuming the Boltzmann distribution $P_{\mathrm{v}}(T)$ at standard room temperature $(298 \mathrm{~K})$.

The Born-Oppenheimer Molecular Dynamics (BOMD) calculations were performed using Density Functional Theory (DFT) along with the $\omega \mathrm{B} 97 \mathrm{XD}^{57}$ exchange-correlation functional and Dunning's correlation-consistent polarization valence basis set of double-zeta quality (cc-pVDZ). ${ }^{46}$ The Bulirsch-Stoer method was used for the integration scheme, ${ }^{58,59}$ along with an integration step size of $0.2 \mathrm{fs}$, and a fifth-order polynomial fit in the integration-correction scheme. The trajectory step size was set to $0.250 \mathrm{au}\left(\mathrm{amu}^{1 / 2}\right.$ bohr), and atomic coordinates were dumped at time intervals of approximately $1 \mathrm{fs}$. Thermalization of the BOMD trajectories to standard room temperature (298 K) was enforced by setting the initial rotational energy from a thermal distribution assuming a symmetric top. The BOMD simulations were performed for a microcanonical (NVE) ensemble and the equilibration time was set to 0.1 ps. Thermalization was checked by monitoring the timedependence of the kinetic energies and potential energies obtained at each point of the computed trajectories. The total runtime was $1.042 \mathrm{ps}$, resulting in the generation of 2000 thermally distorted

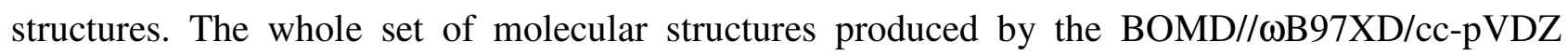


simulations were then used as input geometries in single point DFT calculations employing the B3LYP functional ${ }^{44,45}$ and Dunning's aug-cc-pVDZ basis set. ${ }^{46,47} \mathrm{C}$-shell scripts have been used to

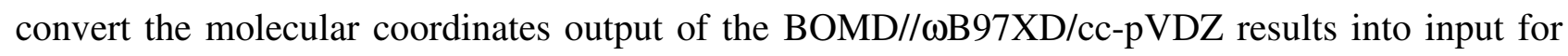
single-point B3LYP/aug-cc-pVDZ calculations of the electronic structure, using the GAUSSIAN09 package of programs, ${ }^{60}$ and to combine results for thermally averaging the electron momentum profiles obtained by means of the MOMAP program by Brion and coworkers. ${ }^{52}$ In addition to these calculations, theoretical momentum distributions were calculated at the B3LYP/aug-cc-pVDZ level for the equilibrium geometry. For making comparisons with experiment, the theoretical momentum profiles were convoluted with the experimental resolution using the procedure by Migdall et al. ${ }^{61}$

\section{RESULTS AND DISCUSSION}

\section{A. Electron Binding Energy Spectrum}

Adamantane belongs to the $\mathrm{T}_{\mathrm{d}}$ point group and its ground state electronic configuration can be written as follows:

$$
(\text { core })^{20}\left(3 \mathrm{a}_{1}\right)^{2}\left(3 \mathrm{t}_{2}\right)^{6}(2 \mathrm{e})^{4}\left(4 \mathrm{t}_{2}\right)^{6}\left(4 \mathrm{a}_{1}\right)^{2}\left(5 \mathrm{a}_{1}\right)^{2}\left(5 \mathrm{t}_{2}\right)^{6}\left(1 \mathrm{t}_{1}\right)^{6}\left(6 \mathrm{t}_{2}\right)^{6}\left(2 \mathrm{t}_{1}\right)^{6}(3 \mathrm{e})^{4}\left(7 \mathrm{t}_{2}\right)^{6} .
$$

In photoelectron spectroscopy (PES) studies of adamantane, ${ }^{12}$ distinct ionization bands have been observed at $E_{\text {bind }} \sim 9.8,11.2,13.5,15.3,17.2$, and $18.8 \mathrm{eV}$, which are ascribed to the $7 \mathrm{t}_{2},\left\{2 \mathrm{t}_{1}+3 \mathrm{e}\right\}$, $\left\{1 \mathrm{t}_{1}+6 \mathrm{t}_{2}\right\},\left\{5 \mathrm{t}_{2}+5 \mathrm{a}_{1}\right\}, 4 \mathrm{a}_{1}$, and $4 \mathrm{t}_{2}$ orbitals, respectively.

Fig. 1 shows the $\Delta \phi$-angle integrated binding energy spectrum of adamantane, which was constructed by plotting the number of true coincidence events as a function of $E_{\text {bind. }}$. Vertical bars indicate the ionization energies reported in the PES studies. To extract contributions from each ionization band, a deconvolution procedure was used. In the procedure a Gaussian curve was assumed for each band, whose energy-position and width were determined from the instrumental energy resolution and the Franck-Condon width estimated from the high energy resolution PES study reported by Boschi et al. ${ }^{12}$ The deconvoluted curves are shown in Fig. 1 as dashed curves and their sum as a solid curve. A similar fitting procedure was applied to a series of binding energy 
spectra at each $\Delta \phi$. Then experimental momentum profiles of the individual ionization bands were produced by plotting the area under the corresponding Gaussian curve against the momentum value. For making a comparison with theory, the $4 \mathrm{a}_{1}$ momentum profile was height normalized to the HAQM calculation at $p=0.18$ a. $\mathrm{u}$. The scaling factor thus obtained was subsequently applied to the individual results so that all the experimental momentum profiles share a common intensity scale.

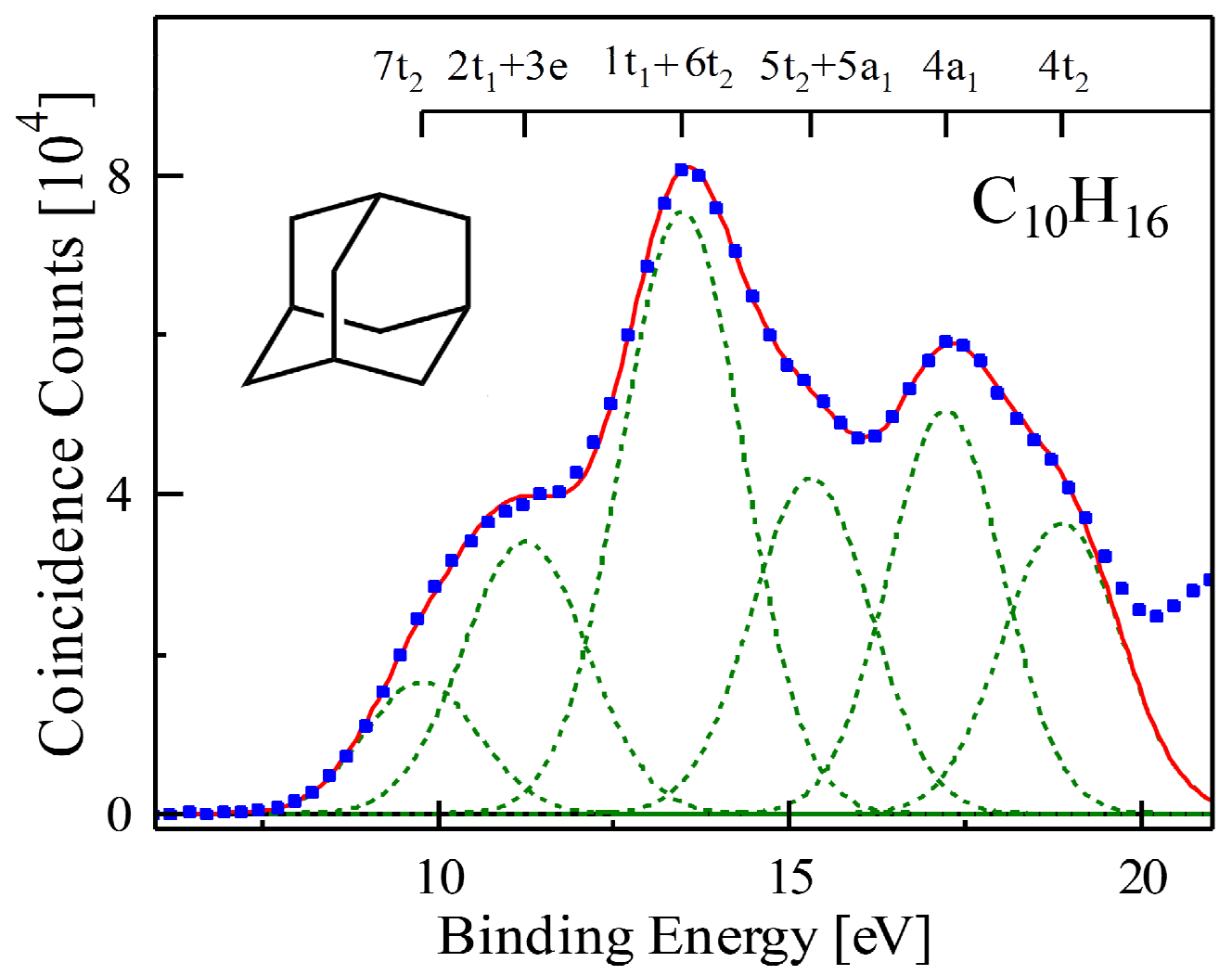

Fig. 1. Momentum-space integrated (e, 2e) outer-valence ionization spectrum of adamantane. The red curve is obtained by summing the Gaussian curves used to deconvolute ionization intensities. The fitting parameters for these Gaussian curves were estimated from a previous PES study [12].

\section{B. Momentum Profiles}

The experimental momentum profiles for the $7 \mathrm{t}_{2},\left\{2 \mathrm{t}_{1}+3 \mathrm{e}\right\},\left\{1 \mathrm{t}_{1}+6 \mathrm{t}_{2}\right\},\left\{5 \mathrm{t}_{2}+5 \mathrm{a}_{1}\right\}$, and $4 \mathrm{a}_{1}$ ionization bands are shown in Fig. 2, along with those reported by Lytviniuk et al., which are digitized from Ref. 39. For comparison, the theoretical momentum profiles calculated at the 
equilibrium geometry are also depicted. Inserted in the figure are theoretical contour plots of the molecular orbitals in position space.
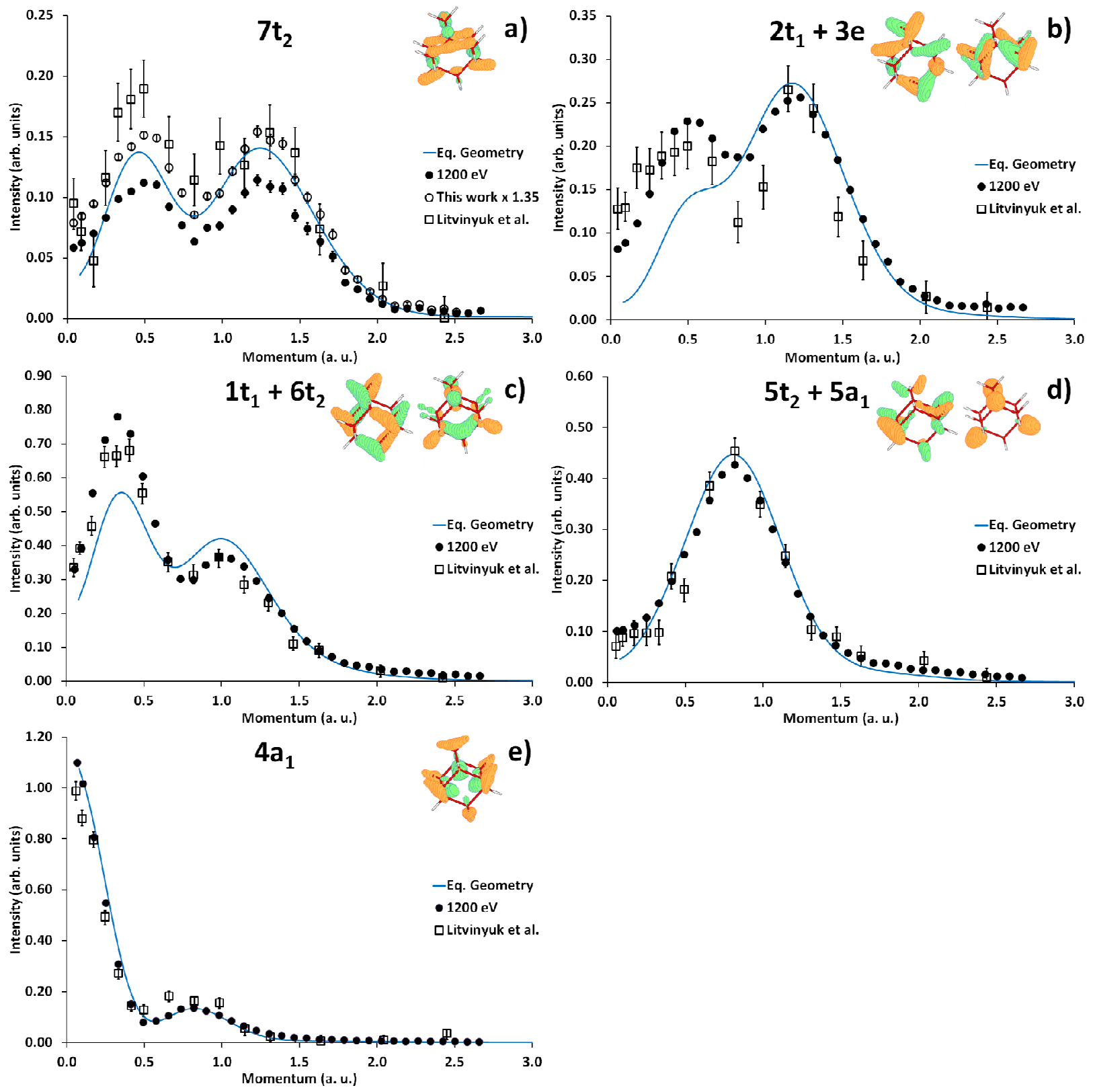

Fig. 2. Experimental and theoretical momentum profiles for the (a) $7 t_{2}$, (b) $\left\{2 t_{1}+3 e\right\}$, (c) $\left\{1 t_{1}+6 t_{2}\right\}$, (d) $\left\{5 \mathrm{t}_{2}+5 \mathrm{a}_{1}\right\}$, and (e) $4 \mathrm{a}_{1}$ ionization bands of adamantane. The solid lines represent the theoretical momentum profiles calculated at the equilibrium geometry. The inserted figures are the theoretical contour plots of molecular orbitals in position space.

It is immediately clear from Fig. 2 that the statistical accuracy of the present data is greatly improved by taking advantage of the high collection efficiency of the energy- and momentum- 
dispersive EMS spectrometer used. Also seen from the figure is that most of the momentum profiles obtained in our measurement are consistent with those measured by Lytviniuk et al. within the error bars. The sole exception is the $7 t_{2}$ band. The present result is about a factor of 1.35 lower in intensity, whilst the shapes of the experimental $7 t_{2}$ momentum profiles are consistent with each other. The origin of the intensity difference may be attributed to deconvolution uncertainties in the fitting procedure, since the adjacent $\left\{2 \mathrm{t}_{1}+3 \mathrm{e}\right\}$ band is only $1.5 \mathrm{eV}$ apart from the $7 \mathrm{t}_{2}$ band and these two ionization bands both exhibit broad Franck-Condon profiles, which considerably overlap with each other, as has been shown by the PES study. ${ }^{12}$

A comparison between experiment and theory shows that all in all, the equilibrium geometry calculation qualitatively reproduces the observed shapes of momentum profiles. In particular, the theoretical prediction for the $4 \mathrm{a}_{1}$ orbital is in excellent agreement with experiment. A closer look at Fig. 2, however, indicates that for the other orbitals the overall agreement between experiment and theory based on equilibrium geometry calculations is not quantitative. Figs. 2(a) - (c) show that for the first three ionization bands, each of which exhibits a double-maximum structure, the equilibrium geometry calculation noticeably underestimates the relative intensity of the maximum at smaller $p$. One may also notice that for the $\left\{5 t_{2}+5 a_{1}\right\}$ band, the theoretical prediction is considerably lower in intensity than the experimental results below $p \sim 0.2$ a.u. Similar tendencies have been observed also by Lytviniuk et al., ${ }^{39}$ but higher statistical accuracy achieved in the present study allows us to make detailed comparisons of experiment with theory. It should be noted that the disagreement with experiment is unlikely to originate from the basis set used, because extension of the basis from aug-cc-pVDZ to augmented correlation-consistent polarization valence-triple-zeta (aug-cc-pVTZ) did not appreciably change the results of the equilibrium geometry calculations.

Based on an argument for d-type orbitals by Brion et al., ${ }^{62}$ Lytviniuk et al. have concluded that the discrepancies between experiment and theory stem from distortion of the incoming and outgoing electron waves participating in the ionization process. The underlying idea of the argument is as follows. Briefly, atomic d-orbitals have gerade symmetry and the zero-gradient 
component of the position-space wave function, which contributes to the $p=0$ component in momentum-space, appears at the nuclear position, where the influences of the target potential to the incoming and outgoing electron waves should be sizable. Hence, the low-momentum component of the d-orbital is substantially affected by distortion of the electron waves from plane waves. It has long been argued that such distorted-wave effects may possibly take place also for molecular orbitals with zero-gradient wave function components in the nuclear and/or internuclear positions. ${ }^{62,63}$ With these considerations in mind, Lytviniuk et al. examined the 3-D visualization of the molecular orbitals and found that the $2 \mathrm{t}_{1}, 3 \mathrm{e}$, and $1 \mathrm{t}_{1}$ orbitals exhibit zero-gradient components in the internuclear (cage) region; they thus attributed the differences between experiment and theory to distorted-wave effects. Nevertheless, the above discussion does not fully account for the observed disagreements. In spite of considerable deviations from theory being observed also for the $7 t_{2}$ and $\left\{5 t_{2}+5 a_{1}\right\}$ bands, there is no zero-gradient component in the cage region for these molecular orbitals. A full understanding of the experimental results therefore requires consideration of additional effects.

The influence of molecular vibration is a possible source of the observed differences, and indeed it gives a rational explanation of most experimental results. To illustrate this, the experimental momentum profiles are compared with the HAQM and BOMD calculations, in which vibrational effects are taken into account. The results for the $7 \mathrm{t}_{2}$, and $\left\{2 \mathrm{t}_{1}+3 \mathrm{e}\right\}$ ionization bands are shown in Fig. 3, and those for the $\left\{1 t_{1}+6 t_{2}\right\},\left\{5 t_{2}+5 a_{1}\right\}$, and $4 a_{1}$ bands in Fig. 4 . In the figures, the HAQM and BOMD momentum profiles are shown as red solid- and black dashed-lines, respectively, while the equilibrium geometry calculations are depicted by blue solid-lines. In addition, the experimental $7 \mathrm{t}_{2}$ momentum profile multiplied by a factor of 1.35 is also shown in Fig. 3(a) for ease of comparison in shape with theory. It is immediately clear from inspecting the figures that the HAQM and BOMD approaches consistently quantify the influence of nuclear dynamics on the momentum profiles. The good accordance of the HAQM calculation, which is based on the harmonic approximation, with the BOMD calculation strongly suggests that the influence of 
vibrational anharmonicity is negligibly small for these momentum profiles, being consistent with our earlier studies on furan ${ }^{35}$ and dimethyl ether. ${ }^{36}$
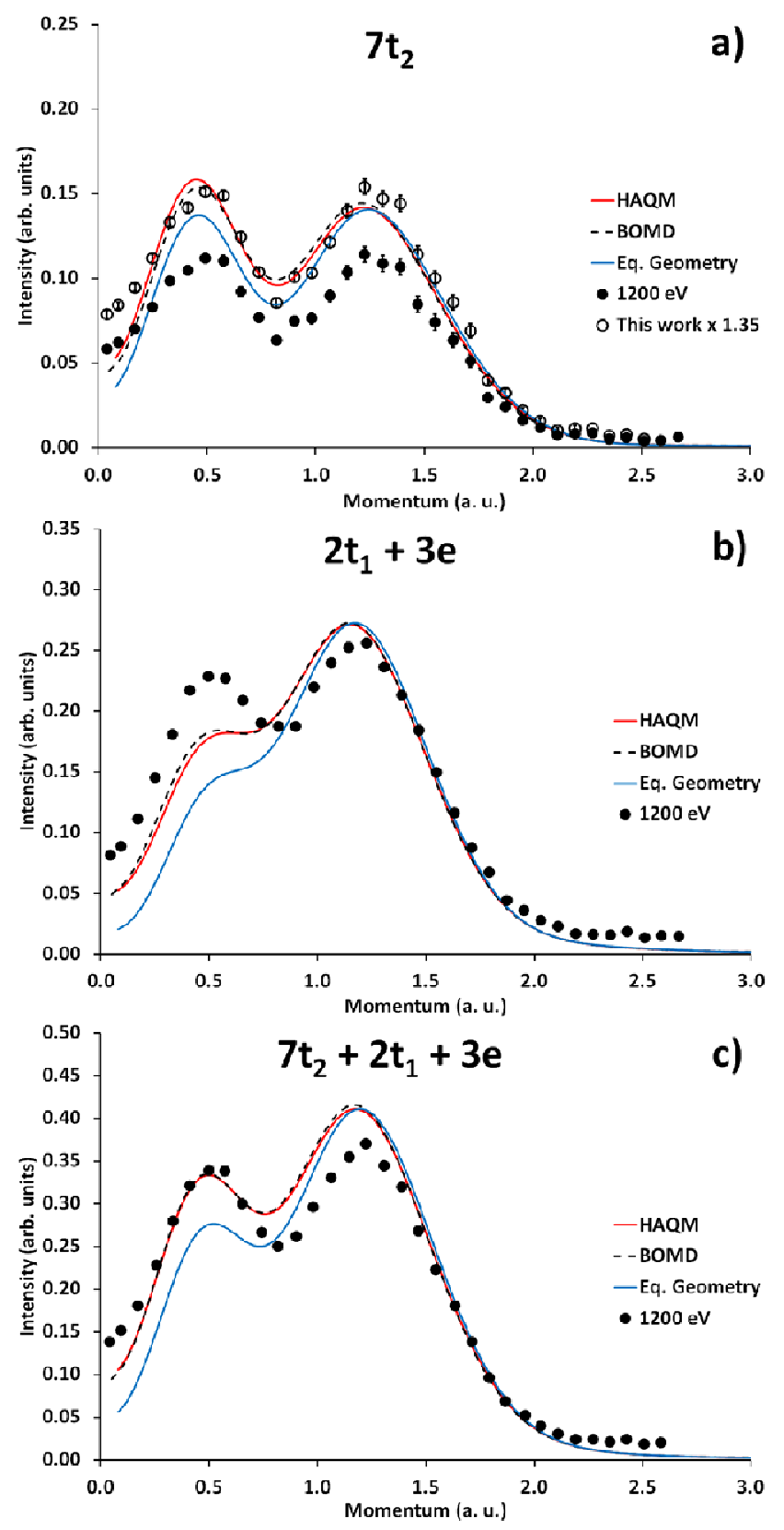

Fig. 3. Comparison between experimental and theoretical momentum profiles for the (a) $7 t_{2}$, (b) $\left\{2 \mathrm{t}_{1}+3 \mathrm{e}\right\}$, and (c) $\left\{7 \mathrm{t}_{2}+2 \mathrm{t}_{1}+3 \mathrm{e}\right\}$ orbitals of adamantane. The red, dashed-, and blue solid-lines represent the HAQM, BOMD, and equilibrium geometry calculations, respectively.

It is evident from Fig. 3 that for the $7 t_{2}$ and $\left\{2 t_{1}+3 e\right\}$ orbitals, taking vibrational effects into account leads to noticeable increase of low-momentum components and as a consequence, improves the agreement with the experimental momentum profiles, though some deviations from 
experiment still remain. As mentioned above, the $7 t_{2}$ and $\left\{2 t_{1}+3 e\right\}$ momentum profiles are likely to suffer from uncertainties of the deconvolution procedure because of the small energy separation between the ionization bands. To remove the possible influence of the deconvolution error, the $7 \mathrm{t}_{2}$ and $\left\{2 \mathrm{t}_{1}+3 \mathrm{e}\right\}$ experimental momentum profiles are plotted in the form of their sum. As can be seen from Fig. 3(c), the HAQM and BOMD calculations are in reasonable agreement with the experimental momentum profile, whilst the equilibrium geometry calculation significantly underestimates the intensity at $p<0.7$ a.u. The findings clearly show that molecular vibration does considerably affect the electron momentum profiles of the $7 t_{2}$ and $\left\{2 t_{1}+3 e\right\}$ orbitals at the low $p$ region, though small discrepancy between experiment and theory still remains at $p=0.7-1.2 \mathrm{a}$. $\mathrm{u}$.

Sizable vibrational effects are observed also for the $\left\{5 t_{2}+5 a_{1}\right\}$ orbitals. It can be seen form Fig. 4(b) that the HAQM and BOMD calculations give higher intensity at small momentum than the equilibrium geometry calculation and satisfactorily reproduce the experiment. The result indicates that the difference between experiment and equilibrium geometry calculation observed for the $\left\{5 \mathrm{t}_{2}+5 \mathrm{a}_{1}\right\}$ band finds its origin into molecular vibrations, and that the HAQM and BOMD approaches both provide good descriptions of vibrational effects on the momentum profile.

Although the influence of molecular vibration gives a rational explanation of most experimental results, this is not the case for the $\left\{1 t_{1}+6 t_{2}\right\}$ ionization band. It can be seen from Fig. $4(a)$ that taking into account vibrational effects does not change the theoretical momentum profile significantly, and all theoretical calculations substantially underestimate the experiment at $p<0.5$ a. u. A plausible explanation of the disagreement is distorted-wave effects. As mentioned above, the $1 t_{1}$ orbital has zero-gradient component in the cage region and hence the influence of distortedwave effects may possibly be appreciable even at small $p$. In principle, the effects can be assessed by examining the incident electron energy dependence of the momentum profile ${ }^{27,28,63}$ however, it is beyond the scope of the present study. In the following we thus focus our attention only to the $7 \mathrm{t}_{2}$, $\left\{2 \mathrm{t}_{1}+3 \mathrm{e}\right\}$, and $\left\{5 \mathrm{t}_{2}+5 \mathrm{a}_{1}\right\}$ orbitals, for which considerable vibrational effects have been observed. 

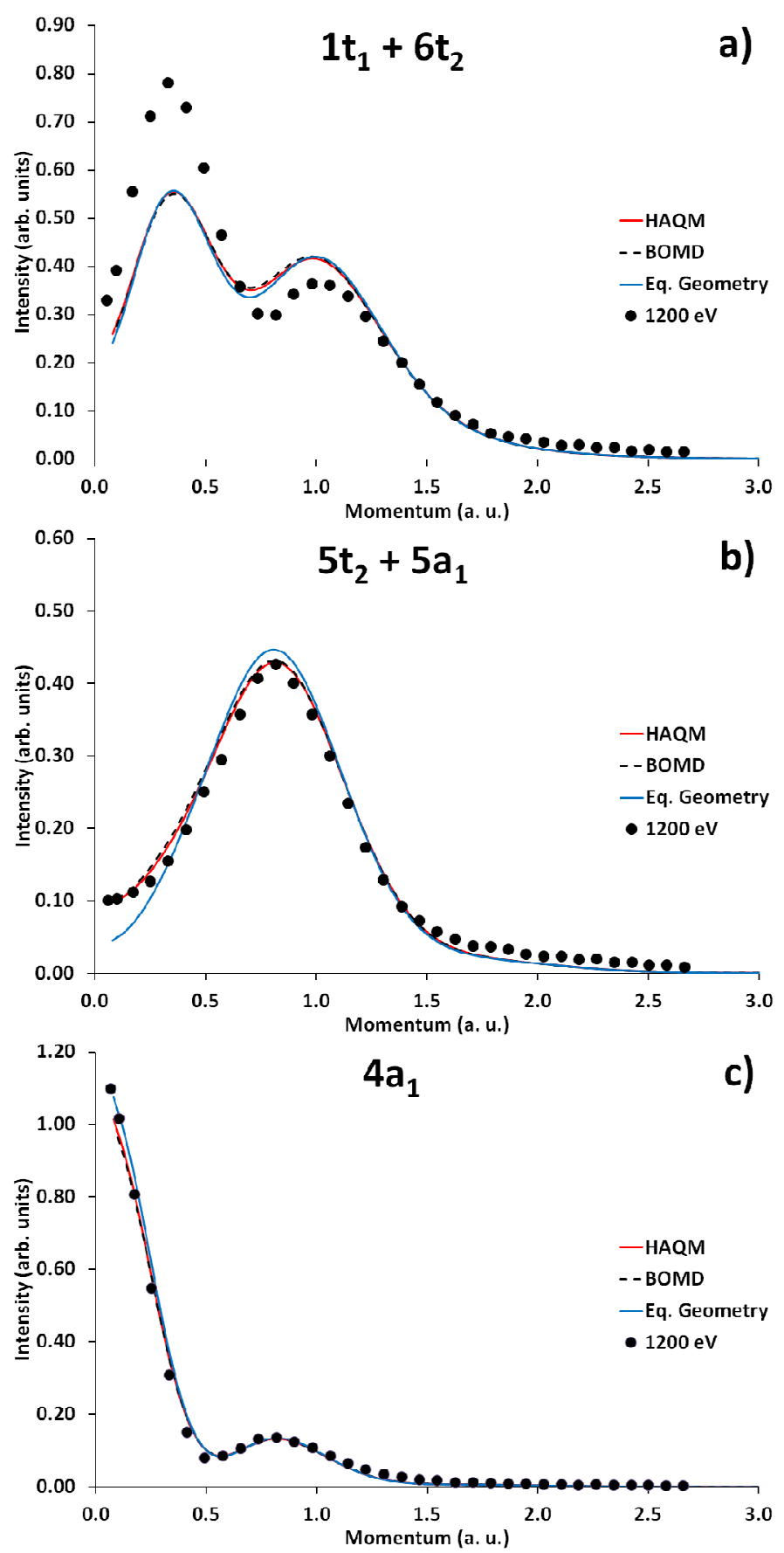

Fig. 4. Comparison between experimental and theoretical momentum profiles for the (a) $\left\{1 t_{1}+6 t_{2}\right\}$, (b) $\left\{5 \mathrm{t}_{2}+5 \mathrm{a}_{1}\right\}$, and (c) $4 \mathrm{a}_{1}$ orbitals of adamantane. The red, dashed-, and blue solid-lines represent the HAQM, BOMD, and equilibrium geometry calculations, respectively.

\section{Contributions from individual vibrational modes}

One of the advantages of the HAQM approach is that vibrational effects on momentum profiles can be discussed in detail by dividing those into contributions from each normal mode. From such 
an analysis, we have found that vibrational effects for the $7 t_{2},\left\{2 t_{1}+3 e\right\}$, and $\left\{5 t_{2}+5 a_{1}\right\}$ orbitals cannot be ascribed to only a few specific normal modes; the contributions are distributed over a number of modes due to the large degrees of vibrations freedom of the adamantane molecule. This is in contrast with findings made recently for smaller molecules, such as $\mathrm{C}_{2} \mathrm{H}_{4}{ }^{30}$ and $\mathrm{CH}_{2} \mathrm{~F}_{2},{ }^{34}$ where only a few normal modes play dominant roles. To facilitate the interpretation of results, the classification of vibrational modes proposed by Jensen ${ }^{50}$ has then been adopted, where each of modes is assigned to one of nine types of motions: $\mathrm{CC}$ stretch, $\mathrm{CH}$ stretch, $\mathrm{CCC}$ bend, $\mathrm{CCC}$ wag, $\mathrm{CH}$ wag, $\mathrm{CH}_{2}$ scissors, $\mathrm{CH}_{2}$ twist, $\mathrm{CH}_{2}$ wag, and $\mathrm{CH}_{2}$ rock motions. The assignment is given in Table I. The contributions from each type of motions to the momentum profiles are displayed in Fig. 5. Here the influences of the $\mathrm{CH}_{2}$ scissors, twist, wag, and rock modes are presented in the form of their sum since these modes all correspond to changing the direction of $\mathrm{CH}$ bonds in the $\mathrm{CH}_{2}$ groups. Similarly, the sum of contributions from the CCC bend and wag modes is presented in the figure.

As can be seen from Fig. 5 (a), the $\mathrm{CH}$ stretching vibrations lead to considerable enhancement of the $p=0$ density component of the $7 \mathrm{t}_{2}$ orbital. Further inspection shows that the $v_{20}, v_{21}$, and $v_{22}$ vibrations play a dominant role at $p \sim 0$, all of which belong to the $\mathrm{T}_{2}$ irreducible representation. Since the momentum space representation of a molecular orbital $\varphi_{\mathrm{f}}(\boldsymbol{p} ; \boldsymbol{Q})$ is uniquely related to the position space wave function $\varphi_{\mathrm{i}}(\boldsymbol{r} ; \boldsymbol{Q})$ through the Dirac-Fourier transform, its amplitude at $p=0$ can be written as

$$
\varphi_{\mathrm{f}}(\boldsymbol{p}=\boldsymbol{0} ; \boldsymbol{Q})=(2 \pi)^{-3 / 2} \int \varphi_{\mathrm{f}}(\boldsymbol{r} ; \boldsymbol{Q}) \mathrm{d} \boldsymbol{r} .
$$

It follows from Eq. (7) that for the molecule at the equilibrium geometry, only totally-symmetric $a_{1}$ orbitals can have non-zero intensity at $p=0$. Although the $7 \mathrm{t}_{2}$ orbital is not totally-symmetric, molecular geometry distortion along the $\mathrm{T}_{2}$ normal coordinates allows its momentum profile to have non-zero intensity at the momentum origin, due to the fact that $T_{2} \otimes T_{2}=A_{1}+E+T_{1}+T_{2}$ in the $\mathrm{T}_{\mathrm{d}}$ point group. This symmetry consideration indicates that the $v_{20}\left(\mathrm{~T}_{2}\right) \sim v_{30}\left(\mathrm{~T}_{2}\right)$ modes may possibly affect the intensity at $p=0$ and the above analysis reveals that among them the relevant 
$\mathrm{CH}$ stretching vibrations mainly contribute to the increase in intensity.

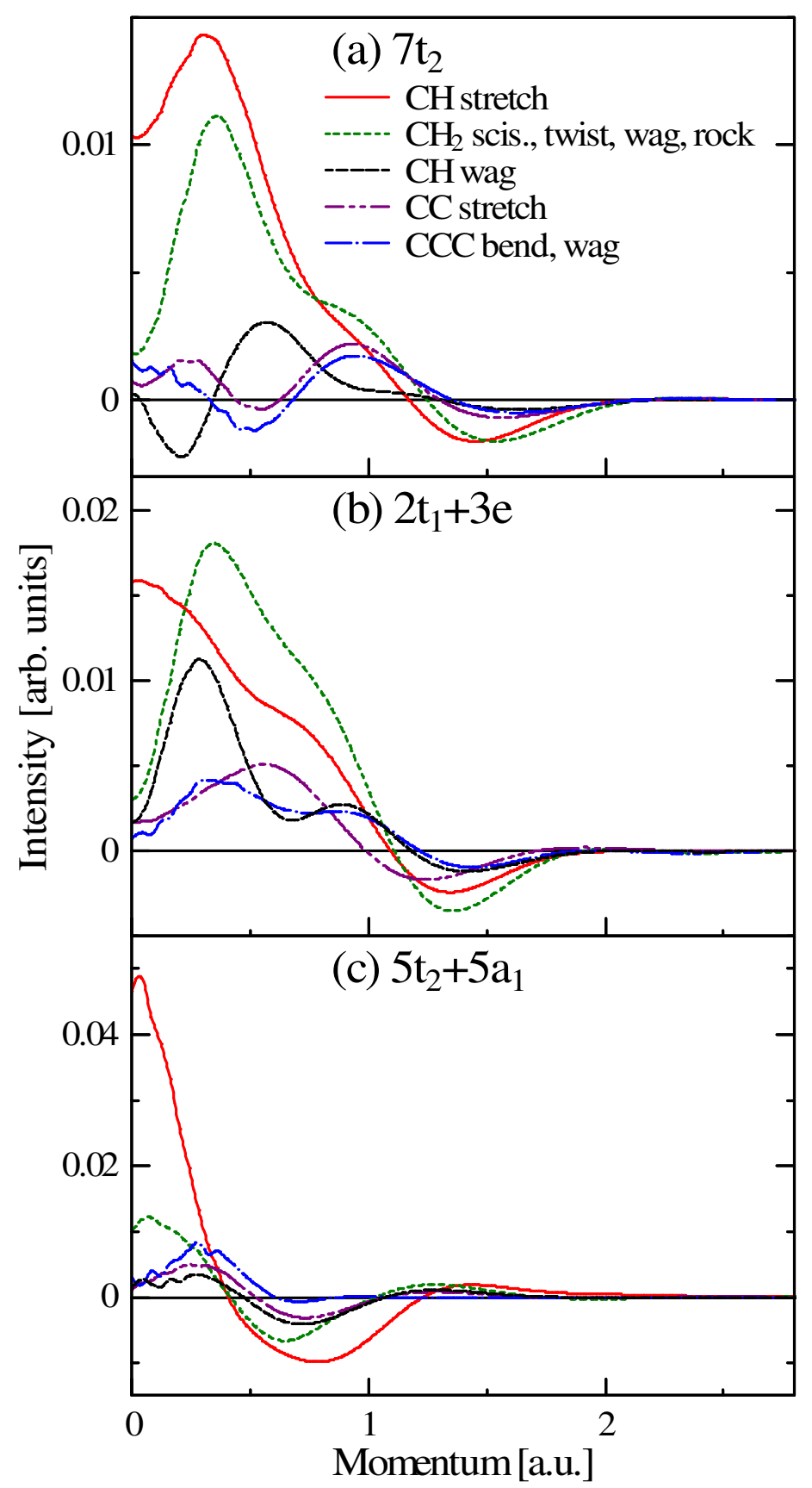

Fig. 5. Contributions from each type of vibrational motion to the momentum profiles of (a) $7 t_{2}$, (b) $\left\{2 \mathrm{t}_{1}+3 \mathrm{e}\right\}$ and (c) $\left\{5 \mathrm{t}_{2}+5 \mathrm{a}_{1}\right\}$ orbitals.

A similar tendency has been observed also for the $\left\{2 \mathrm{t}_{1}+3 \mathrm{e}\right\}$ orbitals. It can be seen from Fig. 5(b) that the $\mathrm{CH}$ stretching vibrations considerably affect the $\left\{2 \mathrm{t}_{1}+3 \mathrm{e}\right\}$ momentum profile at $p \sim 0$. Further analysis has shown that the $v_{13}\left(\mathrm{~T}_{1}\right)$ mode gives the largest contribution to the increase of the magnitude at the momentum origin. It can be deduced from $T_{1} \otimes T_{1}$ being equal to $A_{1}+E+T_{1}$ 
$+\mathrm{T}_{2}$ that the appreciable intensity observed at $p \sim 0$ is mainly due to the distortion of the $2 \mathrm{t}_{1}$ orbital due to the $v_{13} \mathrm{CH}$ stretching vibration.

Also seen from Figs. 5 (a) and (b) is that at around $p=0.4$ a. u., the influences of the $\mathrm{CH}_{2}$ scissors, twist, wag, and rock modes to the $7 \mathrm{t}_{2}$ and $\left\{2 \mathrm{t}_{1}+3 \mathrm{e}\right\}$ momentum profiles are comparable to those from the $\mathrm{CH}$ stretching modes, while the $\mathrm{CC}$ stretch, $\mathrm{CCC}$ bend, and $\mathrm{CCC}$ wag modes play relatively minor roles. For the $\left\{2 \mathrm{t}_{1}+3 \mathrm{e}\right\}$ orbitals, the $\mathrm{CH}$ wag mode also gives a noticeable contribution to the associated momentum profile at the low $p$ region. The results indicate that taking into account contributions from the normal modes associated with $\mathrm{CH}$ bonds is essential for understanding the observed increase in intensity of the $7 \mathrm{t}_{2}$ and $\left\{2 \mathrm{t}_{1}+3 \mathrm{e}\right\}$ momentum profiles at small $p$, being consistent with previous results on ethylene, ${ }^{30} \mathrm{CH}_{2} \mathrm{~F}_{2},{ }^{34}$ furan, ${ }^{35}$ dimethyl ether, ${ }^{36}$ and oxetane $^{38}$ in their contributions to the change of momentum profiles.

We subsequently discuss the vibrational effects on the $\left\{5 t_{2}+5 a_{1}\right\}$ momentum profile. It can be seen from Fig. 5(c) that the change of the momentum profile is mainly due to the $\mathrm{CH}$ stretching vibrations. As can be seen from the figure, the contribution of the $\mathrm{CH}$ stretching modes has a maximum at the momentum origin and rapidly decreases with the increase of $p$. The results can be understood based on the Herzberg-Teller principle. ${ }^{64}$ Within the framework of standard first-order perturbation theory and density functional theory, the target Kohn-Sham orbital at a distorted geometry $\boldsymbol{Q}_{0}+\boldsymbol{Q}^{\prime}$ can be obtained from the orbitals at the equilibrium geometry $\boldsymbol{Q}_{0}$, as follows:

$$
\varphi_{\mathrm{f}}\left(\boldsymbol{r} ; \boldsymbol{Q}_{0}+\boldsymbol{Q}^{\prime}\right)=\varphi_{\mathrm{f}}\left(\boldsymbol{r} ; \boldsymbol{Q}_{0}\right)+\sum_{\mathrm{L}, \mathrm{j} \neq \mathrm{f}} \frac{\left\langle\varphi_{\mathrm{j}}\left|\left[\partial\left(v_{\mathrm{ext}}+v_{\mathrm{Coul}}+v_{\mathrm{XC}}\right) / \partial Q_{\mathrm{L}}\right]_{Q=Q_{0}}\right| \varphi_{\mathrm{f}}\right\rangle}{\varepsilon_{\mathrm{f}}-\varepsilon_{\mathrm{j}}} Q_{\mathrm{L}} \varphi_{\mathrm{j}}\left(\boldsymbol{r} ; \boldsymbol{Q}_{0}\right),
$$

where $v_{\text {ext }}, v_{\text {Coul }}$ and $v_{\mathrm{XC}}$ are the external potential, the Coulomb potential and the non-local exchange-correlation functional, respectively. It can be seen from this Herzberg-Teller expansion that the couplings should effectively take place between states or orbitals with relevant symmetries which give non-vanishing matrix elements involved in the perturbation corrections, and with small energy separations due to the denominators, $\varepsilon_{\mathrm{f}}-\varepsilon_{\mathrm{j}}$. According to group theory, the $5 \mathrm{t}_{2}$ orbital can couple to the neighboring $4 \mathrm{a}_{1}$ orbital through the $v_{20}\left(\mathrm{~T}_{2}\right), v_{21}\left(\mathrm{~T}_{2}\right)$, and $v_{22}\left(\mathrm{~T}_{2}\right) \mathrm{CH}$ stretching modes 
since $T_{2} \otimes A_{1}=T_{2}$, and similarly, the $5 a_{1}$ orbital can couple to the $4 a_{1}$ orbital through the $v_{1}\left(A_{1}\right)$ and $v_{2}\left(\mathrm{~A}_{1}\right)$ modes $\left(\mathrm{A}_{1} \otimes \mathrm{A}_{1}=\mathrm{A}_{1}\right)$. Owing to small energy separation between the $5 \mathrm{t}_{2}\left(5 \mathrm{a}_{1}\right)$ and $4 \mathrm{a}_{1}$ ionization bands, the couplings between the associated orbitals may effectively occur, and hence the $\left\{5 \mathrm{t}_{2}+5 \mathrm{a}_{1}\right\}$ ionization band borrows its intensity from the $4 \mathrm{a}_{1}$ band. Because the $4 \mathrm{a}_{1}$ momentum profile has an intense, sharp maximum at $p=0$, coupling to this orbital results in the increase in intensity at small $p$.

\section{Jahn-Teller effect on the $7 t_{2}$ orbital}

When an electron is removed from the triply degenerate $\left(7 \mathrm{t}_{2}\right)^{6}$ orbital a $\mathrm{C}_{10} \mathrm{H}_{16}{ }^{+}$cation in ${ }^{2} \mathrm{~T}_{2}$ electronic ground state is obtained. This is subject to a Jahn-Teller (JT) distortion from its original $\mathrm{T}_{\mathrm{d}}$ point group, which leads to a structure having a $\mathrm{C}_{3 \mathrm{v}}$ symmetry in a ${ }^{2} \mathrm{~A}_{1}$ electronic ground state with a new electronic configuration $(12 \mathrm{e})^{4}\left(12 \mathrm{a}_{1}\right)^{1}$.

This carbocation and the JT effect on adamantane have been thoroughly investigated from a theoretical and experimental point of view ${ }^{9,10,65-71}$. The presence of this molecular species has been inferred from the strong asymmetric broadening in the ionization band of the $7 \mathrm{t}_{2}$ orbital from both photoelectron (PES) $)^{18,72-74}$ and Penning ionization (PIES) spectroscopy ${ }^{18}$. Direct evidence for a Jahn-Teller distortion of the adamantane cation is given by infrared spectroscopy. ${ }^{70}$.

Upon keeping in mind these evidences, it is worth evaluating the extent to which momentum profiles can be affected by Jahn-Teller distortions and how these can be detected with EMS. The results of Fig. 6 show a comparison between momentum profiles calculated at the B3LYP/aug-ccpVDZ level for the equilibrium geometry and profiles computed for the $\mathrm{C}_{3 \mathrm{v}}$ molecular structure of the cation ${ }^{66,67,70}$, along with the individual components for both the $12 \mathrm{e}$ and $12 \mathrm{a}_{1}$ orbitals. 


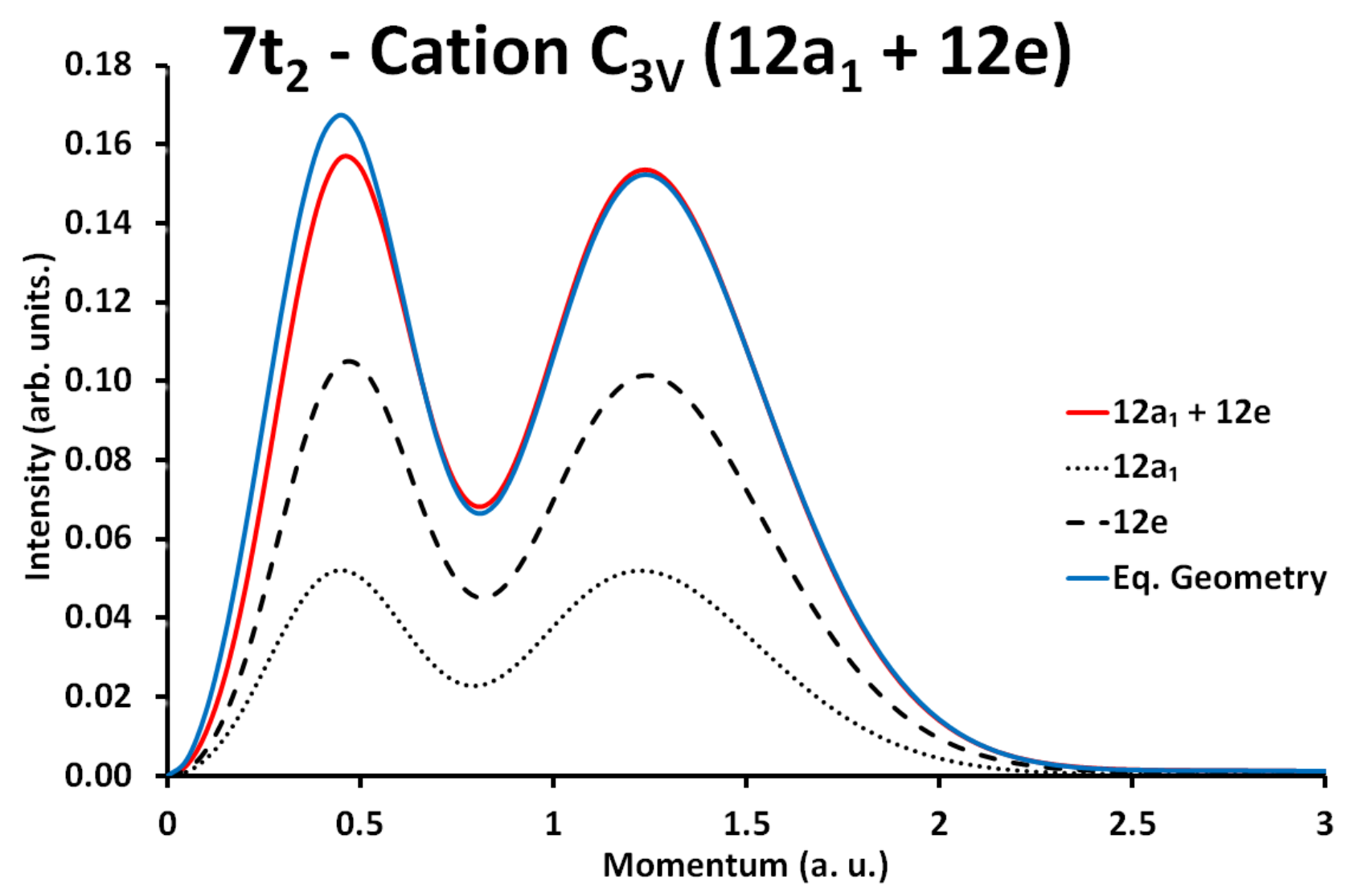

Fig. 6. Comparison between the momentum profiles of the HOMO (7t $\mathrm{t}_{2}$ of $\mathrm{C}_{10} \mathrm{H}_{16}$ and that one of the JT distorted structure $\mathrm{C}_{10} \mathrm{H}_{16}{ }^{+}\left(\mathrm{C}_{3 \mathrm{v}}\right)$ including its individual components $12 \mathrm{a}_{1}$ and $12 \mathrm{e}$.

This comparison shows that the Jahn-Teller distortion due to ionization of the HOMO $\left(7 \mathrm{t}_{2}\right)$ of adamantane does not have a significant influence on the momentum profile of the corresponding $\left(12 \mathrm{a}_{1}\right.$ and $\left.12 \mathrm{e}\right)$ orbitals of the cation, both in shape and intensity. Only a small decrease of intensity is observed at $p<0.5$ a.u. when comparing results for the relaxed molecular structure of the cation with that for equilibrium geometry. This limited influence is due to the fact that the molecular target is large so that the structural relaxation effects induced by ionization are limited ${ }^{70}$, although the ionized $7 \mathrm{t}_{2}$ orbital has a strong $\sigma_{\mathrm{C}-\mathrm{C}}$ bonding character.

Nevertheless this observation demonstrates the possibility to study JT effects on this molecule by means of future EMS experiments with higher resolution, analogously to previous studies of cyclopropane and $\mathrm{NH}_{3},{ }^{75,76}$. Higher energy resolution will be required to measure the individual $12 \mathrm{a}_{1}$ and $12 \mathrm{e}$ momentum profiles, which considerably differ from one another, considering that the 
energy separation between the $12 \mathrm{a}_{1}$ and $12 \mathrm{e}$ orbitals of the cation does not exceed $0.55 \mathrm{eV}$.

\section{Conclusions}

In this study, we have investigated the influence of molecular vibrations on the electron momentum distributions of the outer valence orbitals of the smallest diamondoid, adamantane. The EMS experiment on the molecule has been conducted at $E_{0}=1.2 \mathrm{keV}$. Furthermore, theoretical momentum profiles with vibrational effects being involved have been calculated by means of the HAQM and BOMD approaches. In spite of their intrinsic differences and despite the complex nature of the vibrational structure of the chosen target, both approaches provide consistent results and give overall quantitative insights into the results of the newly performed EMS experiment with improved statistics, except for the $\left\{1 \mathrm{t}_{1}+6 \mathrm{t}_{2}\right\}$ ionization band. Comparisons between experiment and theory has shown that taking into account vibrational effects lead to appreciable enhancement of the low $p$ component of the $7 \mathrm{t}_{2},\left\{2 \mathrm{t}_{1}+3 \mathrm{e}\right\}$ and $\left\{5 \mathrm{t}_{2}+5 \mathrm{a}_{1}\right\}$ momentum profiles and considerably improve the agreement with experiment. Further analysis based on the HAQM approach has revealed the significant role of vibrational modes associated with the $\mathrm{CH}$ bonds, in line with previous results on molecules with several $\mathrm{CH}$ bonds $\mathrm{s}^{30,34-36,38}$ in their contribution to the change of momentum profiles.

\section{Acknowledgment}

This work has been supported by the FWO_Vlaanderen, the Flemish branch of the Belgian National Science Foundation, the "Bijzonder Onderzoeks Fonds" of Hasselt University, and the Japanese Ministry of Education, Culture, Sports, Science and Technology, Grant-in-Aids for Scientific Research (A) (No. 25248002) and (B) (No. 15H03761) and Challenging Exploratory Research (Nos. 25620006 and 15K13615). The computational resources and services used in this work were provided by the VSC (Flemish Supercomputer Center), funded by the Research Foundation - Flanders (FWO) and the Flemish Government - department EWI. 


\section{References}

${ }^{1}$ G. A. Mansoori, P. L. B. de Araujo and E. S. de Araujo, Diamondoid Molecules With Applications in Biomedicine, Materials Science, Nanotechnology \& Petroleum Science (World Scientific, Singapore, 2012).

${ }^{2}$ H. Schwertfeger, A. A. Fokin, and P. R. Schreiner, Angew. Chem. Int. Ed. 47, 1022 - 1036 (2008).

${ }^{3}$ L. Landt, K. Klünder, J. E. Dahl, R. M. K. Carlson, T. Möller, and C. Bostedt, Phys. Rev. Lett. 103, 047402 (2009).

${ }^{4}$ T. Zimmermann, R. Richter, A. Knecht, A. A. Fokin, T. V. Koso, L. V. Chernish, P. A. Gunchenko, P. R. Schreiner, T. Möller, and T. Rander, J. Chem. Phys. 139, 084310 (2013).

${ }^{5}$ R. Richter, M. I. S. Röhr, T. Zimmermann, J. Petersen, C. Heidrich, R. Rahner, T. Möller, J. E. Dahl, R. M. K. Carlson, R. Mitric, T. Rander, and A. Merli, Phys. Chem. Chem. Phys. 17, 4739 (2015).

${ }^{6}$ F. Marsusi, J. Sabbaghzadeh, and N. D. Drummond, Phys. Rev. B 84, 245315 (2011).

${ }^{7}$ C. E. Patrick and F. Giustino, Nat. Commun. 4, 2006 (2013).

${ }^{8}$ S. Banerjee and P. Saalfrank, Phys. Chem. Chem. Phys. 16, 144 (2014).

${ }^{9}$ T. Demján, M. Vörös, M. Palummo, and A. Gali, J. Chem. Phys. 141, 064308 (2014).

${ }^{10}$ A. Gali, T. Demján, M. Vörös, G. Thiering, E. Cannuccia, and A. Marini, Nat. Commun. 7, 11327 (2016).

${ }^{11}$ W. Schmidt, Tetrahedron 29, 2129 (1973).

${ }^{12}$ R. Boschi, W. Schmidt, R.J. Suffolk, B.T. Wilkins, H.J. Lempka, J.N.A. Ridyard, J. Electron Spectrosc. Relat. Phenom. 2, 377 (1973).

${ }^{13}$ Q. Y. Shang and E. R. Bernstein, J. Chem. Phys. 100, 8265 (1994). 
${ }^{14}$ V. Galasso, J. Mol. Struct. (Theochem) 336, 47 (1995).

${ }^{15}$ M. S. Deleuze and J. Delhalle, Int. J. Quantum Chem. 60, 1505 (1996).

${ }^{16}$ V. Galasso, J. Mol. Struct. (Theochem) 528, 171 (2000).

${ }^{17}$ D. V. Korolkov and O. V. Sizova, Int. J. Quantum Chem. 88, 606 (2002).

${ }^{18}$ S. X. Tian, N. Kishimoto, and K. Ohno, J. Phys. Chem. A 106, 6541 (2002).

${ }^{19}$ G. Yan, N. R. Brinkmann, and H. F. Schaefer III, J. Phys. Chem. A 107, 9479 (2003).

${ }^{20}$ A. J. Lu, B. C. Pan and J. G. Han, Phys. Rev. B 72, 035447 (2005).

${ }^{21}$ H. Sabzyan and B. Saed, Struct. Chem. 25, 1207 (2014).

${ }^{22}$ I. E. McCarthy and E. Weigold, Phys. Rep. 27, 275 (1976).

${ }^{23}$ C. E. Brion, Int. J. Quantum Chem. 29, 1397 (1986).

${ }^{24}$ K. T. Leung, in Theoretical Models of Chemical Bonding, Part 3, ed. by Z. B. Maksic, SpringerVerlag, Berlin, pp. 339-386 (1991).

${ }^{25}$ I. E. McCarthy and E. Weigold, Rep. Prog. Phys. 54, 789 (1991).

${ }^{26}$ M. A. Coplan, J. H. Moore, and J. P. Doering, Rev. Mod. Phys. 66, 985 (1994).

${ }^{27}$ E. Weigold and I. E. McCarthy, Electron Momentum Spectroscopy (Kluwer Academic/Plenum Publishers, New York, 1999).

${ }^{28}$ M. Takahashi, Bull. Chem. Soc. Jpn. 82, 751 (2009).

${ }^{29}$ Y. R. Miao, J. K. Deng, and C. G. Ning, J. Chem. Phys. 136, 124302 (2012).

${ }^{30}$ N. Watanabe, M. Yamazaki, and M. Takahashi, J. Chem. Phys. 137, 114301 (2012).

${ }^{31}$ B. Hajgató, F. Morini, and M. S. Deleuze, Theor. Chem. Acc. 131, 1244 (2012). 
${ }^{32}$ Y. R. Miao, J. M. Li, J. K. Deng, and C. G. Ning, J. Electron Spectrosc. Relat. Phenom. 193, 1 (2014).

${ }^{33}$ J. Yang, X. Shan, Z. Zhang, Y. Tang, M. Zhao, and X. J. Chen, J. Phys. Chem. A 118, 11780 (2014).

${ }^{34}$ N. Watanabe, M. Yamazaki, and M. Takahashi, J. Chem. Phys. 141, 244314 (2014).

${ }^{35}$ F. Morini, M. S. Deleuze, N. Watanabe, and M. Takahashi, J. Chem. Phys. 142, 094308 (2015).

${ }^{36}$ F. Morini, N. Watanabe, M. Kojima, M. S. Deleuze, and M. Takahashi, J. Chem. Phys. 143, 134309 (2015).

${ }^{37}$ N. Watanabe, M. Yamazaki and M. Takahashi, J. Electron Spectrosc. Relat. Phenom. 209, 78 (2016).

${ }^{38}$ Y. Tang, X. Shan, J. Yang, S. Niu, Z. Zhang, N. Watanabe, M. Yamazaki, Masahiko Takahashi, and X. J. Chen, J. Phys. Chem. A 120, 6855 (2016).

${ }^{39}$ I. V. Litvinyuk, Y. Zheng, and C. E. Brion, Chem. Phys. 253, 41 (2000).

${ }^{40}$ M. Takahashi, N. Watanabe, Y. Khajuria, K. Nakayama, Y. Udagawa, and J. H. D. Eland, J. Electron Spectrosc. Relat. Phenom. 141, 83 (2004).

${ }^{41}$ T. Helgaker, E. Uggerud, and H. J. A. Jensen, Chem. Phys. Lett. 173, 145 (1990).

${ }^{42}$ E. Uggerud and T. Helgaker, J. Am. Chem. Soc. 114, 4265 (1992).

${ }^{43}$ K. Bolton, W. L. Hase, and G. H. Peslherbe, Modern Methods for Multidimensional Dynamics Computation in Chemistry, edited by D. L. Thompson (World Scientific, Singapore, 1998), p. 143.

${ }^{44}$ A. D. Becke, J. Chem. Phys. 98, 5648 (1993).

${ }^{45}$ C. Lee, W. Yang, and R. G. Parr, Phys. Rev. B 37, 785 (1998). 
${ }^{46}$ T. H. Dunning, Jr., J. Chem. Phys. 90, 1007 (1989).

${ }^{47}$ R. A. Kendall, T. H. Dunning, and R. J. Harrisson, J. Chem. Phys. 96, 6796 (1992).

${ }^{48}$ M. J. Frisch et al., GAUSSIAN 03, Revision A.1, Gaussian, Inc., Pittsburgh, PA, 2003.

${ }^{49}$ G. Ramachandran and S. Manogaran, J. Mol. Struct. (Theochem) 766, 125 (2006).

${ }^{50}$ J. O. Jensen, Spectrochim. Acta A 60, 1895 (2004).

${ }^{51}$ P. Duffy, D. P. Chong, M. E. Casida, and D. R. Salahub, Phys. Rev. A 50, 4707 (1994).

${ }^{52}$ A. O. Bawagan, C. E. Brion, E. R. Davidson, and D. Feller, Chem. Phys. 113, 19 (1987).

${ }^{53}$ M. S. Deleuze, L. S. Cederbaum, J. Chem. Phys., 105, 7583 (1996).

${ }^{54}$ M. S. Deleuze and L. S. Cederbaum, Adv. Quantum Chem., 35, 77 (1999).

${ }^{55}$ S. Knippenberg, K. L. Nixon, H. Mackenzie-Ross, Michael J. Brunger, F. Wang, M. S. Deleuze, J.-P. François, D. A. Winkler, J. Phys. Chem. A, 109, 9324 (2005).

${ }^{56}$ Y. R. Huang, S. Knippenberg, B. Hajgató, J.-P. François, J. K. Deng, and M. S. Deleuze, J. Phys. Chem. A 111, 5879 (2007).

${ }^{57}$ J. D. Chai and M. Head-Gordon, Phys. Chem. Chem. Phys. 10, 6615 (2008).

${ }^{58}$ R. Bulirsch and J. Stoer, Introduction to Numerical Analysis (Springer-Verlag, New York, NY, 1991).

${ }^{59}$ W. H. Press, B. P. Flannery, S. A. Teukolsky, and W. T. Vetterling, Richardson Extrapolation and the Bulirsch-Stoer Method. Numerical Recipes in FORTRAN: The Art of Scientific Computing, 2nd ed. (Cambridge University Press, Cambridge, England, 1992), pp. 718-725.

${ }^{60}$ M. J. Frisch, G. W. Trucks, H. B. Schlegel, G. E. Scuseria, M. A. Robb, J. R. Cheeseman, G. Scalmani, V. Barone, B. Mennucci, G. A. Petersson et al., GAUSSIAN 09, Revision B.01, Gaussian, Inc., Wallingford, CT, 2009. 
${ }^{61}$ J. N. Migdall, M. A. Coplan, D. S. Hench, J. H. Moore, J. A. Tossell, V. H. Smith, Jr., and J. W. Liu, Chem. Phys. 57, 141 (1981).

${ }^{62}$ C.E. Brion, Y. Zheng, J. Rolke, J. J. Neville, I. E. McCarthy, J. Wang, J. Phys. B: At. Mol. Opt. Phys. 31, L223 (1998).

${ }^{63}$ X. G. Ren, C. G. Ning, J. K. Deng, S. F. Zhang, G. L. Su, F. Huang, and G. Q. Li, Phys. Rev. Lett. 94, 163201 (2005).

${ }^{64}$ G. Herzberg and E. Teller, Z. Phys. Chem. B21, 410 (1933).

${ }^{65}$ A. A. Fokin, P. A. Gunchenko, S. A. Peleshanko, P. von Ragué Schleyer, and P. R. Schreiner, Eur. J. Org. Chem. 1999, 855 (1999).

${ }^{66}$ A. A. Fokin, P. R. Schreiner, P. A. Gunchenko, S. A. Peleshanko, T. E. Shubina, S. D. Isaev, P. V. Tarasenko, N. I. Kulik, H.-M. Schiebel, and A. G. Yurchenko, J. Am. Chem. Soc. 122, 7317 (2000).

${ }^{67}$ A. A. Fokin, and P. R. Schreiner, Chem. Rev. 102, 1551 (2002).

${ }^{68}$ C. E. Patrick and F. Giustino, Nat. Commun. 4, 2006 (2013)

${ }^{69}$ A. A. Novikovskii, P. A. Gunchenko, P. G. Prikhodchenko, Y. A. Serguchev, P. R. Schreiner, and A. A. Fokin, Rus. J. Org. Chem. 47, 1276 (2011).

${ }^{70}$ A. Patzer, M. Schütz, T. Möller, and Otto Dopfer, Angew. Chem. Int. Ed. 51, 4925 (2012).

${ }^{71}$ M. E. Crestoni and S. Fornarini, Angew. Chem. Int. Ed. 51, 7373 (2012).

${ }^{72}$ J. W. Raymonda, J. Chem. Phys. 56, 3912 (1972).

${ }^{73}$ S. D. Worley, G. D. Mateescu, C. McFarlan, R. C. Fort, C. F. Sheley, J. Am. Chem. Soc. 95, 7580 (1973).

${ }^{74}$ W. Schmidt, Tetrahedron 1973, 29, 2129

${ }^{75}$ Z. J. Li, X. J. Chen, X. Shan, T. Liu, and K. Z. Xu, J. Chem. Phys. 130, 054302 (2009).

${ }^{76}$ J. S. Zhu, Y. R. Miao, J. K. Deng, and C. G. Ning, J. Chem. Phys. 137, 174305 (2012). 
TABLE I. Harmonic vibrational frequencies of adamantane.

\begin{tabular}{|c|c|c|c|}
\hline \multirow{2}{*}{ Normal mode } & \multicolumn{2}{|c|}{ Frequencies $\left(\mathrm{cm}^{-1}\right)$} & \multirow{2}{*}{$\begin{array}{l}\text { Type of } \\
\text { motion }^{c}\end{array}$} \\
\hline & Theory $^{\mathrm{a}}$ & Experiment $^{\mathrm{b}}$ & \\
\hline \multicolumn{4}{|l|}{$\mathrm{A}_{1}$} \\
\hline$v_{1}$ & 3044.1 & 2944 & $\mathrm{CH}$ stretch \\
\hline$v_{2}$ & 3009.8 & 2913 & $\mathrm{CH}$ stretch \\
\hline$v_{3}$ & 1514.9 & 1472 & $\mathrm{CH}_{2}$ scissors \\
\hline$v_{4}$ & 1058.3 & - & CCC bend \\
\hline$v_{5}$ & 757.2 & 756 & $\mathrm{CC}$ stretch \\
\hline \multicolumn{4}{|l|}{$\mathrm{A}_{2}$} \\
\hline$v_{6}$ & 1116.2 & - & $\mathrm{CH}_{2}$ twist \\
\hline \multicolumn{4}{|l|}{$\mathrm{E}$} \\
\hline$v_{7}$ & 3012.4 & 2900 & $\mathrm{CH}$ stretch \\
\hline$v_{8}$ & 1476.4 & 1439 & $\mathrm{CH}_{2}$ scissors \\
\hline$v_{9}$ & 1397.7 & 1370 & CH wag \\
\hline$v_{10}$ & 1222.4 & 1217 & $\mathrm{CH}_{2}$ twist \\
\hline$v_{11}$ & 918.4 & 906 & $\mathrm{CC}$ stretch \\
\hline$v_{12}$ & 418.1 & 402 & $\mathrm{CCC}$ bend \\
\hline \multicolumn{4}{|l|}{$\mathrm{T}_{1}$} \\
\hline$v_{13}$ & 3050.5 & - & $\mathrm{CH}$ stretch \\
\hline$v_{14}$ & 1338.0 & 1312 & $\mathrm{CH}_{2}$ wag \\
\hline$v_{15}$ & 1308.9 & - & $\mathrm{CH}_{2}$ twist \\
\hline$v_{16}$ & 1113.4 & 1105 & $\mathrm{CH}$ wag \\
\hline$v_{17}$ & 1052.5 & - & CC stretch \\
\hline$v_{18}$ & 898.4 & - & $\mathrm{CH}_{2}$ rock \\
\hline$v_{19}$ & 343.9 & 335 & CCC wag \\
\hline \multicolumn{4}{|l|}{$\mathrm{T}_{2}$} \\
\hline$v_{20}$ & 3055.8 & 2950 & $\mathrm{CH}$ stretch \\
\hline$v_{21}$ & 3033.8 & 2944 & $\mathrm{CH}$ stretch \\
\hline$v_{22}$ & 3011.4 & 2910 & $\mathrm{CH}$ stretch \\
\hline$v_{23}$ & 1490.9 & 1455 & $\mathrm{CH}_{2}$ scissors \\
\hline$v_{24}$ & 1376.7 & 1359 & $\mathrm{CH}_{2}$ wag \\
\hline$v_{25}$ & 1333.6 & 1310 & $\mathrm{CH}$ wag \\
\hline$v_{26}$ & 1115.3 & 1101 & $\mathrm{CH}_{2}$ rock \\
\hline$v_{27}$ & 978.7 & 970 & CC stretch \\
\hline$v_{28}$ & 815.0 & 804 & CC stretch \\
\hline$v_{29}$ & 658.1 & 638 & CCC bend \\
\hline$v_{30}$ & 461.9 & 444 & CCC wag \\
\hline
\end{tabular}

${ }^{a}$ This work (B3LYP/aug-cc-pVDZ).

${ }^{\mathrm{b}}$ See ref. 49.

${ }^{\mathrm{c}}$ See ref. 50. 